\title{
The demographic features, clinicopathologic characteristics, treatment outcome and disease-specific prognostic factors of solitary fibrous tumor: a population-based analysis
}

\author{
Alimujiang Wushou ${ }^{1,2}$, Yi-Zhou Jiang ${ }^{1,2}$, Yi-Rong Liu' ${ }^{1,2}$ and Zhi-Ming Shao ${ }^{1,2}$ \\ ${ }^{1}$ Department of Breast Surgery, Key Laboratory of Breast Cancer in Shanghai, Fudan University Shanghai Cancer Center, \\ Fudan University, Shanghai, China \\ 2 Department of Oncology, Shanghai Medical College, Fudan University, Shanghai, China \\ Correspondence to: Zhi-Ming Shao, email: zhimingshao@yahoo.com orfd-can@hotmail.com
}

Keywords: solitary fibrous tumor, SEER analysis

Received: August 09, $2015 \quad$ Accepted: October 06, $2015 \quad$ Published: October 19, 2015

This is an open-access article distributed under the terms of the Creative Commons Attribution License, which permits unrestricted use, distribution, and reproduction in any medium, provided the original author and source are credited.

\section{ABSTRACT}

Background: Solitary fibrous tumor's (SFT) demographic features, clinicopathologic characteristics, treatment outcome and disease-specific prognostic factors were unexplored comprehensively.

Methods: SEER program was used to identify patients diagnosed with SFT from 1973 to 2012. Overall collected data were analyzed by using the SPSS 18.0.

Results: In total, 804 cases were found including 613 cases with SFT-specific mortality and 801 patients were analyzed for overall survival (OS). The 3-year disease free survival (DFS), 5-year DFS and 10-year DFS were $73.3 \%, 65.7 \%$ and $53.3 \%$. The 3-year OS, 5-year OS and 10 -year OS were $71.9 \%, 63.3 \%$ and $47.3 \%$. In the multivariate survival analysis, the age $>51$ years (hazard ratio [HR] $=1.851$ for DFS, $P=0.024$ and $H R=1.652$ for OS, $P=0.033$; Reference $[R e f] \leq 51$ years for DFS and $\leq$ 53 years for OS), SEER stage metastasized tumor $(H R=4.269$ for $D F S, P=0.000$ and HR $=2.905$ for OS, $P=\mathbf{0 . 0 2 8}$, Ref - localized + regional tumor), pathologic grade III + IV (HR $=2.734$ for DFS, $P=0.001$ and HR $=2.585$ for $0 S, P=0.000$, Ref - grade $I$ + II) were adversely associated with DFS and OS. In addition, surgery was favorably associated with DFS (HR $=0.217, P=0.045$, Ref - surgery + radiotherapy).

Conclusions: The surgery was an independent prognostic factor for DFS. The patient's age, SEER stage and pathologic grade were SFT-specific independent prognostic indicators for DFS and OS.

\section{INTRODUCTION}

Soft tissue sarcomas represent a heterogeneous group of mesenchymal tumors with variable clinical behavior and prognosis [1]. As a result, the treatment modalities of soft tissue sarcoma are increasingly subtypespecific. There are big challenges for the oncologist in treating patients with rare subtypes of soft tissue sarcoma, since adequate instructive data from clinical trials or even coherent studies are not available to guide rigorous evidence-based treatment [2]. Hemangiopericytoma was firstly named by Stout and Murray in 1942. It derives from pericytes around capillaries and postcapillary venules, thus, this tumor can be found in any sites of human body that contain capillaries [3]. The term "hemangiopericytoma" has been retired; tumors diagnosed as hemangiopericytoma in the past are now called solitary fibrous tumor (SFT) [4]. SFT is one of the most commonly misdiagnosed soft tissue sarcomas in clinic because of its unspecific initial clinical presentation. Moreover, accurate pathologic diagnosis of SFT is also a challenge, because up to $15 \%$ of all soft tissue sarcomas can have SFT-like pathologic characteristics [5].

Our previous study investigated head and neck (HN) SFTs and found that a treatment protocol emphasizing the surgical removal of the tumor as the first-line treatment, tumor size $>5.0 \mathrm{~cm}$, poor pathologic differentiation, deep tumor location and non-surgical treatment were independent adverse prognostic factors for HN SFTs $[6,7]$. 
Currently, only case reports and retrospective series have been reported with relatively small of study population from variety of tumor location. Given the rarity of this tumor, information is still sporadic regarding their unique demographic, clinicopathologic, prognostic and biologic characteristics. Oncologists around the globe have limited management experience, resulting in heterogeneity of treatment paradigms and prognosis.

We speculate that a large nationwide populationbased patient cohort may provide an opportunity to evaluate trends in demographic features, clinical presentation, patient characteristics, diagnosis, treatment modalities and prognostic factors of SFT. To better characterize unique demographic features, clinicopathologic characteristics, treatment outcome and prognostic factors of SFT, we carried out a comprehensive analysis of all patients with SFT registered in the Surveillance, Epidemiology, and End Results (SEER) public-access database collected from various geographic areas in the United States from 1973 to 2012.

\section{RESULTS}

\section{The demographic and clinicopathologic characteristics of 613 SFT patients with disease- specific survival status}

A total of 613 patients with diagnosis of SFT were found in the SEER database with SFT-specific mortality spanning from 1973 to 2012 . The median follow-up time was 86.8 (range, 1-478) months. Gender distribution was almost equal between males and females (45.4\% and $54.6 \%$, respectively) and a peak incidence occurred during the fifth decade of life. Majority of SFT occurred in white people. About half of the tumors registration came from the Pacific coast. Approximately, one third of SFT cases originated from thoracic, abdominal and pelvic cavity (TAPC) and one-half of SFT cases were classified as SEER stage regional tumor. Surgery was the most common treatment modality and $49.4 \%$ of cases were treated with surgery alone. Most of the tumors were pathologically unclassified SFTs and they accounted for nearly $70 \%$ of cases. The baseline characteristics of 613 SFT patients are summarized in Table 1.

\section{Disease free survival (DFS) and cox proportional hazards regression analysis}

Kaplan-Meier analysis was performed for time-toevent analysis for DFS. The 3-year DFS, 5-year DFS, 10-year DFS, 15-year DFS were 73.3\%, 65.7\%, 53.3\% and $45.9 \%$, respectively. Significant DFS differences were found depending on age $(P=0.000)$, marital status $(P=0.000)$, SEER stage $(P=0.006)$, tumor location $(P$ $=0.000)$, treatment modality $(P=0.000)$ and pathologic grade $(P=0.001)$ (Figure 1$)$. A Cox proportional hazard model was performed to identify prognostic variables for DFS. In the univariate Cox regression analysis, the age $>$ 51 years $(P=0.000 ; \mathrm{HR}=2.341 ; \leq 51$ years - as reference [Ref]), SEER stage distant metastasized tumor $(P=0.012$; $\mathrm{HR}=1.532$; localized + regional tumor - as Ref $)$ and pathologic grade III + IV $(P=0.002 ; \mathrm{HR}=2.210$; grade I + II - as Ref) were associated with worse DFS. SFT from TAPC $(P=0.009 ; \mathrm{HR}=0.412$; central nerve system $[\mathrm{CNS}]$ - as Ref $)$ and surgery alone $(P=0.000 ; \mathrm{HR}=$ 0.353 ; surgery with radiotherapy - as Ref) were associated with favorable DFS. In the multivariate Cox regression analysis, the age $(P=0.024 ; \mathrm{HR}=1.851 ; \leq 51$ years as Ref), SEER stage $(P=0.000 ; \mathrm{HR}=4.269$ for distant metastasized tumor; localized + regional tumor - as Ref), treatment modalities $(P=0.045$; HR $=0.217$ for surgery alone; surgery $+\mathrm{RT}$ - as Ref) and pathologic grade $(P=$ $0.001 ; \mathrm{HR}=2.734$ for grade III + IV; grade I + II - as Ref) were independent prognostic variables for DFS. Details of the Cox proportional hazards regression analysis are shown in Tables 2 and 3.

\section{The demographic and clinicopathologic characteristics of 801 SFT patients with overall survival (OS) status}

From 1973 to 2012, there were 804 consecutive registered patients with SFT in the SEER database. Three patients were excluded for survival analysis, due to lack of treatment and follow-up data. The 801 patients included 361 male patients and 440 female patients, with a male-tofemale ratio of $0.82: 1$. Their ages ranged from 1 month to $85+$ years, with a median of 53 years (Supplementary Figure 1). More than $80 \%$ of SFT cases occurred in white people and only $8.8 \%$ in black people. Half of the cases came from the Pacific coast, 220 cases from east, 128 cases from the northern plains and 53 cases from southwest. In total, $35.3 \%$ of tumors originated from TAPC, $24.5 \%$ from CNS, $19.9 \%$ from $\mathrm{HN}$ region and $16.2 \%$ from extremity. According to SEER stage classification, 48.3\%, 18.9\% and $13.9 \%$ patients were classified as localized, regional and distant metastasized SFTs. $87.6 \%$ patients treated with surgery included treatment modalities. Only 244 SFT patients had defined pathologic grade data. The median follow-up period was 85.6 (range, 1-478) months. The baseline characteristics of 801 SFT patients with OS status are presented in Table 1. 

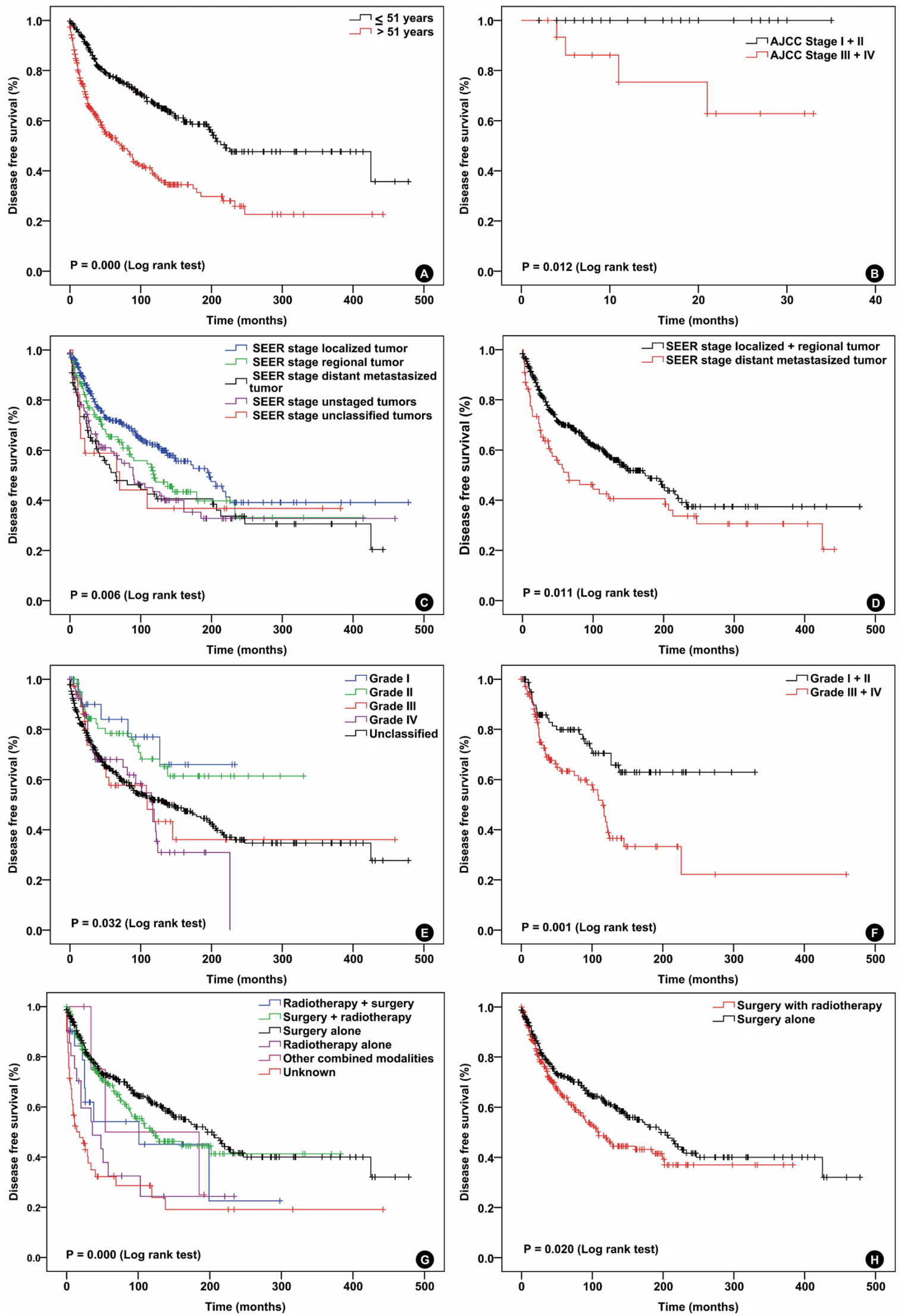

Figure 1: Disease free survival curves of patients with solitary fibrous tumor compared according to A. age, B. AJCC stage, C. and D. SEER stage, E. and F. pathologic grade, G. and H. treatment modalities. Log-rank test was used to compare curves, and significance $(P$ value $)$ is shown on each panel. 
Table 1: The baseline characteristics of SFT patients in the SEER database

\begin{tabular}{|c|c|c|c|c|c|c|c|c|c|}
\hline \multirow{2}{*}{\multicolumn{2}{|c|}{$\begin{array}{l}\text { Demographic and clinicopathologic } \\
\text { parameters }\end{array}$}} & \multicolumn{4}{|l|}{ DFS } & \multicolumn{4}{|l|}{ OS } \\
\hline & & Alive & Dead & Total & $P$-value & Alive & Dead & Total & $P$-value \\
\hline \multirow{2}{*}{ Gender } & Female & 183 & 152 & 335 & \multirow{2}{*}{0.584} & 196 & 244 & 440 & \multirow{2}{*}{0.205} \\
\hline & Male & 158 & 120 & 278 & & 177 & 184 & 361 & \\
\hline \multirow{4}{*}{ Age } & $\leq 51$ years & 204 & 107 & 311 & \multirow{2}{*}{0.000} & - & - & - & - \\
\hline & $>51$ years & 137 & 165 & 302 & & - & - & - & - \\
\hline & $\leq 53$ years & - & - & - & - & 226 & 177 & 403 & \multirow{2}{*}{0.000} \\
\hline & $>53$ years & - & - & - & - & 147 & 251 & 398 & \\
\hline \multirow{3}{*}{ Race } & White & 267 & 229 & 496 & \multirow{3}{*}{0.178} & 293 & 361 & 654 & \multirow{3}{*}{0.033} \\
\hline & Black & 31 & 19 & 50 & & 34 & 37 & 71 & \\
\hline & Others & 43 & 24 & 67 & & 46 & 30 & 76 & \\
\hline \multirow{4}{*}{$\begin{array}{l}\text { Marital } \\
\text { status }\end{array}$} & Single & 100 & 48 & 148 & \multirow{4}{*}{0.000} & 104 & 73 & 177 & \multirow{4}{*}{0.000} \\
\hline & Married & 189 & 154 & 343 & & 211 & 235 & 446 & \\
\hline & Divorced & 15 & 29 & 44 & & 15 & 48 & 63 & \\
\hline & Others & 37 & 41 & 78 & & 43 & 72 & 115 & \\
\hline \multirow{4}{*}{$\begin{array}{l}\text { CHSDA } \\
\text { Region }\end{array}$} & East & 122 & 54 & 176 & \multirow{4}{*}{0.000} & 130 & 90 & 220 & \multirow{4}{*}{0.000} \\
\hline & Northern plain & 29 & 63 & 92 & & 31 & 97 & 128 & \\
\hline & Pacific coast & 179 & 128 & 307 & & 199 & 201 & 400 & \\
\hline & Southwest & 11 & 27 & 38 & & 14 & 39 & 53 & \\
\hline \multirow{5}{*}{$\begin{array}{l}\text { Tumor } \\
\text { Locations }\end{array}$} & Central nerve system & 97 & 60 & 157 & \multirow{5}{*}{0.000} & 105 & 91 & 196 & \multirow{5}{*}{0.001} \\
\hline & Head and neck & 83 & 34 & 117 & & 91 & 69 & 160 & \\
\hline & TAPC & 100 & 109 & 209 & & 109 & 174 & 283 & \\
\hline & Extremity & 50 & 57 & 107 & & 55 & 75 & 130 & \\
\hline & Other location & 11 & 12 & 23 & & 13 & 19 & 32 & \\
\hline \multirow{2}{*}{ AJCC Stage } & $\mathrm{I}+\mathrm{II}$ & 26 & 0 & 26 & \multirow{2}{*}{0.007} & 33 & 0 & 33 & 0001 \\
\hline & $\mathrm{III}+\mathrm{IV}$ & 12 & 4 & 16 & & 16 & 6 & 22 & 0.001 \\
\hline & Localized & 194 & 109 & 303 & & 210 & 177 & 387 & \\
\hline & Regional & 72 & 58 & 130 & & 77 & 74 & 151 & \\
\hline SEER stage & Distant metastasized & 31 & 47 & 78 & 0.000 & 36 & 75 & 111 & 0.000 \\
\hline & Unstaged & 37 & 48 & 85 & & 43 & 88 & 131 & \\
\hline & Unclassified & 7 & 10 & 17 & & 7 & 14 & 21 & \\
\hline & Radiotherapy + surgery & 10 & 10 & 20 & & 10 & 15 & 25 & \\
\hline & Surgery + radiotherapy & 126 & 86 & 212 & & 139 & 130 & 259 & \\
\hline Trutmon & Surgery alone & 181 & 122 & 303 & 0000 & 194 & 217 & 411 & 0009 \\
\hline Heatintit & Radiotherapy alone & 7 & 14 & 21 & 0.000 & 7 & 22 & 29 & 0.002 \\
\hline & OCM & 2 & 3 & 5 & & 3 & 4 & 7 & \\
\hline & Unknown & 15 & 37 & 52 & & 20 & 50 & 70 & \\
\hline & Grade I & 18 & 5 & 23 & & 20 & 11 & 31 & \\
\hline & Grade II & 44 & 18 & 62 & & 45 & 40 & 85 & \\
\hline $\begin{array}{l}\text { Pathologic } \\
\text { grade }\end{array}$ & Grade III & 19 & 18 & 37 & 0.014 & 21 & 24 & 45 & 0.138 \\
\hline & Grade IV & 37 & 30 & 67 & & 41 & 42 & 83 & \\
\hline & Unclassified & 223 & 201 & 424 & & 246 & 311 & 557 & \\
\hline
\end{tabular}

Abbreviations: SEER, surveillance, epidemiology, and end results; SFT, solitary fibrous tumor; DFS, disease free survival; OS, overall survival; CHSDA, contract health service delivery areas; TAPC, thoracic, abdominal and pelvic cavity; OCM, Others combined modalities; AJCC, american joint committee on cancer.

\section{OS and cox proportional hazards regression analysis}

The 3-year OS, 5-year OS, 10-year OS and 15year OS rates were $71.9 \%, 63.3 \%, 47.3 \%$ and $38 \%$, respectively. Significant OS differences were identified depending on age $(P=0.000)$, marital status $(P=0.000)$, CHSDA region $(P=0.006)$, tumor location $(P=0.006)$, AJCC stage $(P=0.003)$, SEER stage $(P=0.027)$, treatment modality $(P=0.000)$ and pathologic grade $(P=$ 

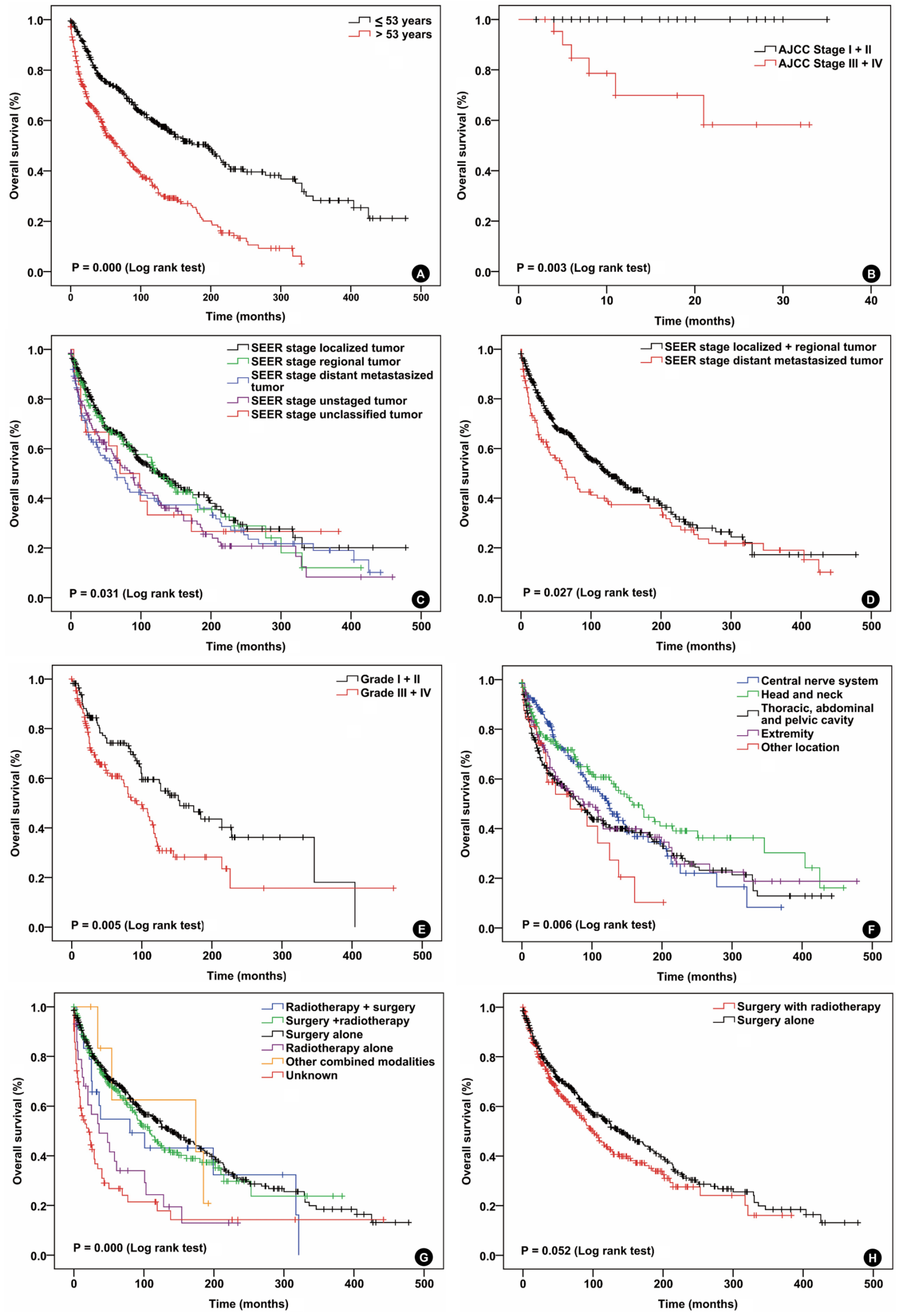

Figure 2: Overall survival curves of patients with solitary fibrous tumor compared according to A. age, B. AJCC stage, C. and D. SEER stage, E. pathologic grade, F. tumor location G. and H. treatment modalities. Log-rank test was used to compare curves, and significance $(P$ value) is shown on each panel. 
Table 2: Univariate Cox regression analysis of characteristics associated with DFS and OS status

\begin{tabular}{|c|c|c|c|c|c|}
\hline \multirow{2}{*}{\multicolumn{2}{|c|}{ Parameters }} & \multicolumn{2}{|l|}{ DFS } & \multicolumn{2}{|l|}{ OS } \\
\hline & & \multirow{2}{*}{\begin{tabular}{|l|} 
HR (95\% CI) \\
1.00 Reference
\end{tabular}} & \multirow{3}{*}{$\begin{array}{l}P \text {-value } \\
0.786\end{array}$} & \multirow{2}{*}{$\frac{\text { HR (95\% CI) }}{1.00 \text { Reference }}$} & \multirow{3}{*}{$\begin{array}{l}\boldsymbol{P} \text {-value } \\
0.834\end{array}$} \\
\hline \multirow{2}{*}{ Gender } & Female & & & & \\
\hline & Male & $1.034(0.813-1.315)$ & & $0.980(0.809-1.186)$ & \\
\hline \multirow{2}{*}{ Age } & $\leq 51$ years & 1.00 Reference & \multirow{2}{*}{0.000} & 1.00 Reference & \multirow{2}{*}{0.000} \\
\hline & $>51$ years & $2.341(1.829-2.996)$ & & $2.253(1.850-2.742)$ & \\
\hline \multirow{3}{*}{ Race } & White & 1.00 Reference & & 1.00 Reference & \\
\hline & Black & $1.353(0.888-2.061)$ & 0.159 & $1.430(0.985-2.075)$ & 0.060 \\
\hline & Others & $1.317(0.721-2.406)$ & 0.370 & $1.662(1.026-2.691)$ & 0.039 \\
\hline \multirow{2}{*}{ Marital status } & Married & 1.00 Reference & & 1.00 Reference & \\
\hline & Other status & $1.006(0.970-1.280)$ & 0.964 & $1.049(0.867-1.269)$ & 0.626 \\
\hline \multirow{4}{*}{$\begin{array}{l}\text { CHSDA } \\
\text { Region }\end{array}$} & East & 1.00 Reference & & 1.00 Reference & \\
\hline & Northern plain & $0.411(0.258-0.654)$ & 0.000 & $0.628(0.431-0.914)$ & 0.015 \\
\hline & Pacific coast & $0.925(0.589-1.453)$ & 0.736 & $0.996(0.687-1.445)$ & 0.985 \\
\hline & Southwest & $0.570(0.376-0.864)$ & 0.008 & $0.761(0.540-1.073)$ & 0.119 \\
\hline \multirow{5}{*}{ Locations } & Central nerve system & 1.00 Reference & & 1.00 Reference & \\
\hline & Head and neck & $0.537(0.288-1.001)$ & 0.050 & $0.579(0.353-0.950)$ & 0.031 \\
\hline & TAPC & $0.412(0.213-0.798)$ & 0.009 & $0.493(0.296-0.822)$ & 0.007 \\
\hline & Extremity & $0.789(0.434-1.435)$ & 0.438 & $0.755(0.470-1.215)$ & 0.248 \\
\hline & Other location & $0.800(0.429-1.493)$ & 0.484 & $0.696(0.420-1.154)$ & 0.160 \\
\hline \multirow{2}{*}{ AJCC Stage } & Stage I + II & 1.00 Reference & \multirow{2}{*}{0.271} & 1.00 Reference & \multirow{2}{*}{$\begin{array}{l}0.182 \\
0.160\end{array}$} \\
\hline & Stage III + IV & $11.035(0.024-549.7)$ & & $0.010(0.000-8.658)$ & \\
\hline \multirow{2}{*}{ SEER stage } & Localized + regional & 1.00 Reference & \multirow{2}{*}{0.012} & 1.00 Reference & \multirow{2}{*}{0.028} \\
\hline & Distant metastasized & $1.532(1.100-2.134)$ & & $1.343(1.032-1.749)$ & \\
\hline \multirow{6}{*}{ Treatment } & Radiotherapy + surgery & 1.00 Reference & & 1.00 Reference & \\
\hline & Surgery + radiotherapy & $0.489(0.242-0.992)$ & 0.047 & $0.450(0.252-0.803)$ & 0.007 \\
\hline & Surgery alone & $0.353(0.237-0.526)$ & 0.000 & $0.365(0.261-0.508)$ & 0.000 \\
\hline & Radiotherapy alone & $0.294(0.200-0.431)$ & 0.000 & $0.333(0.244-0.454)$ & 0.000 \\
\hline & OCM & $0.742(0.398-1.384)$ & 0.348 & $0.751(0.454-1.242)$ & 0.264 \\
\hline & Unknown & $0.436(0.134-1.422)$ & 0.169 & $0.361(0.130-1.000)$ & 0.050 \\
\hline \multirow{2}{*}{ Treatment } & Surgery with radiotherapy & 1.00 Reference & \multirow{2}{*}{0.021} & 1.00 Reference & \multirow{2}{*}{0.053} \\
\hline & Surgery alone & $0.738(0.570-0.955)$ & & $1.226(0.997-1.506)$ & \\
\hline \multirow{2}{*}{$\begin{array}{l}\text { Pathologic } \\
\text { grade }\end{array}$} & Grade I + II & 1.00 Reference & \multirow{2}{*}{0.002} & 1.00 Reference & \multirow{2}{*}{0.005} \\
\hline & Grade III + IV & $2.210(1.338-3.649)$ & & $1.694(1.169-2.455)$ & \\
\hline
\end{tabular}

Abbreviations: SEER, surveillance, epidemiology, and end results; SFT, solitary fibrous tumor; DFS, disease free survival; OS, overall survival; HR, hazard ratio; CI, confidence interval; CHSDA, contract health service delivery areas; TAPC, thoracic, abdominal and pelvic cavity; OCM, Others combined modalities; AJCC, american joint committee on cancer.

0.005) (Figure 2 and Supplementary Figure 2). Univariate and multivariate survival analysis utilizing the Cox regression model were performed on the 801 SFT patients. In the univariate Cox regression analysis, the age $>53$ years $(P=0.000 ; \mathrm{HR}=2.253, \leq 53$ years - as $\mathrm{Ref})$, distant metastasized tumor $(P=0.028$; HR $=1.343$; localized + regional tumor - as Ref) and pathologic grade III + IV ( $P$ $=0.005 ; \mathrm{HR}=1.694$; Grade I + II - as Ref) were adversely associated with OS. SFT from TAPC $(P=0.007, \mathrm{HR}=$ 0.493; CNS- as Ref $)$, surgery $(P=0.000, \mathrm{HR}=0.365$; RT + surgery - as Ref) were favorably associated with OS. The age $(P=0.033, \mathrm{HR}=1.652$ for $>53$ years $; \leq 53$ years - as Ref $),$ SEER stage $(P=0.028 ; \mathrm{HR}=2.905$ for distant metastasized tumor; localized + regional tumor - as Ref) and pathologic grade $(P=0.000 ; \mathrm{HR}=2.585$ for grade III + IV; grade I + II - as Ref) were independent prognostic variables for OS (Tables 2 and 3 ).

\section{DISCUSSION}

According to the current investigation, incidence rate in gender is almost equal for DFS and slightly higher in female for OS. There is no statistically significant predominance in any gender. In survival analysis, remarkable difference was neither identified in DFS, nor in OS $(P=0.786$ and $P=0.833$, respectively, Supplementary Figure 2). In most of the previously published case series, the typical age at presentation ranged from the third to the 
Table 3: Multivariate Cox regression analysis of characteristics associated with DFS and OS status

\begin{tabular}{|c|c|c|c|c|c|}
\hline \multirow{2}{*}{\multicolumn{2}{|c|}{ Parameters }} & \multicolumn{2}{|l|}{ DFS } & \multicolumn{2}{|l|}{ OS } \\
\hline & & \multirow{2}{*}{$\begin{array}{l}\mathrm{HR}(95 \% \mathrm{CI}) \\
1.00 \text { Reference }\end{array}$} & \multirow{3}{*}{$\begin{array}{l}\boldsymbol{P} \text {-value } \\
0.024\end{array}$} & \multirow{2}{*}{$\frac{\mathrm{HR}(95 \% \mathrm{CI})}{1.00 \text { Reference }}$} & \multirow{3}{*}{\begin{tabular}{|l}
$\boldsymbol{P}$-value \\
0.033
\end{tabular}} \\
\hline \multirow{2}{*}{ Age } & $\leq 51$ years & & & & \\
\hline & $>51$ years & $1.851(1.083-3.165)$ & & $1.652(1.042-2.618)$ & \\
\hline \multirow{2}{*}{ SEER stage } & Localized + regional & 1.00 Reference & \multirow{2}{*}{0.000} & 1.00 Reference & \multirow{2}{*}{0.028} \\
\hline & Metastasized & 4.269 (2.144-8.499) & & $2.905(1.673-5.043)$ & \\
\hline \multirow{4}{*}{$\begin{array}{l}\text { Treatment } \\
\text { modalities }\end{array}$} & Radiotherapy + surgery & 1.00 Reference & & 1.00 Reference & \\
\hline & Surgery + radiotherapy & $0.493(0.129-0.719)$ & 0.412 & $0.880(0.170-4.572)$ & 0.879 \\
\hline & Surgery alone & $0.217(0.156-0.747)$ & 0.045 & $0.811(0.158-2.364)$ & 0.476 \\
\hline & Radiotherapy alone & $0.229(0.375-2.994)$ & 0.055 & $0.586(0.154-2.225)$ & 0.432 \\
\hline \multirow{2}{*}{$\begin{array}{l}\text { Pathologic } \\
\text { grade }\end{array}$} & Grade I + II & 1.00 Reference & \multirow{2}{*}{0.001} & 1.00 Reference & \multirow{2}{*}{0.000} \\
\hline & Grade III + IV & $2.734(1.517-4.925)$ & & $2.585(1.556-4.284)$ & \\
\hline
\end{tabular}

Abbreviations: DFS, disease free survival; OS, overall survival; HR, hazard ratio; CI, confidence interval; SEER, surveillance, epidemiology, and end results.

fifth decades [8-10]. In this series, SFT most frequently occurs during the fifth decades of life (Supplementary Figure 2). The majority of investigation about SFT are based on single-center experience and mostly include no more than 30 50 cases. Given their small sample size, those investigations are often not sufficiently powerful to detect small differences in survival analysis according to common demographic factors such as age, gender and race. In our current investigation, patient's age, gender and race were analyzed as categorical variables in both univariate and multivariate survival analyses. The results demonstrate that apart from the age, no survival difference in DFS or OS was found to be associated with race or gender (Table 2 and Supplementary Figure 2).

Up to date, more than two thousand studies are available about SFT in the PubMed. SFT is reported from a variety of tumor locations. In accordance with previous reports, SFT occurred at any age-period and any site of the body in present series (Supplementary Figure 1). To better characterize, we categorized the tumor location into five groups (CNS, HN, TAPC, extremity and other location), although there are many other possibilities to categorize the tumor location. In this categorization, significant survival differences were found, where HN and TAPC SFTs were favorably associated with DFS (Ref - CNS) in univariate survival analysis. However, tumor location was not an independent prognostic indicator in multivariate survival analysis for neither DFS nor OS. Well consisting with our current results, previously, investigators found survival difference between CNS and extra-CNS site SFTs, and no association was found between the primary tumor site and survival [11].

The TNM/AJCC staging system plays important roles and it helps the oncologist in making a treatment protocol and evaluating a prognosis of cancer [12]. In this series, one of the most incomplete data was TNM/ AJCC staging information and only 55 patients' data were available. However, important findings emerged. There was a significant survival difference in AJCC stage
I + II versus AJCC stage III + IV. The small number of patients with AJCC stage in the Cox model did not permit a multivariate survival analysis for independent prognostic factor. In our previous report, we also failed to perform the AJCC stage in multivariate survival analysis of HN SFT due to the incomplete staging data. As far as we know, there aren't any report regarding TNM/AJCC stage identified as an independent prognostic variable for SFT. Nevertheless, detailed SEER stage data was available in this study and it was an independent prognostic indicator not only for DFS but also for OS.

Whether the SFT patients can benefit from RT is most widely investigated hot topic, especially in the CNS SFTs [13-17]. One of the main interests of this study is to hopefully confirm the role of RT in SFT treatment. The RT for SFT has been widely used alone or combined with surgery as part of the management algorithm for several decades. Until now, the role of RT in SFT treatment is still controversial. Lately, investigators analyzed 227 CNS SFT cases in the SEER database during the years of 2000-2009 and confirmed a survival benefit for patients treated with surgery in combination with adjuvant RT, while the effect was not appreciated with surgery alone. In another series, investigators found that treatment protocol of combined adjuvant RT with surgery seemed to hinder tumor progression, but had no effect on OS [18]. The addition of adjuvant RT to surgery improved the local recurrence, but did not increase overall survival of patients with HN SFTs [7, 19]. Owing to lack of data about recurrence, we were unable to confirm whether the RT could decrease the local recurrence in current series. We categorized treatment modalities including surgery and RT as surgery with RT group and compared its treatment outcome with surgery alone. The results demonstrate that surgery alone group had higher DFS and OS, and statistical significance was only found in DFS (Figure $1 \mathrm{H}$ and Figure $2 \mathrm{H}$ ). It is noteworthy that mostly advanced stage SFT patients received RT pre- or postoperatively, and RT was added to early stage SFT once coming across 
common adverse prognostic factors after surgery such as high pathologic grade and unconfirmed surgical margins $[6,7,10]$. As retrospective study, it is almost impossible to make a standard survival comparison between patients treated with surgery alone and surgery with RT. Because those patients did not even have similar TNM/AJCC stage. Therefore, it is difficult to draw a firm conclusion that SFT patients cannot benefit from RT. Multi-center prospective controlled clinical studies are necessary to further confirm the role of RT in SFT treatment.

We acknowledge the limitations that come with our investigation. In the SEER database, data on important factors such as TNM/AJCC stage and pathologic grade are incomplete. Establishment of the role of chemotherapy is another important issue. A notable limitation of the SEER database is that there is no chemotherapy information available for analysis. In addition, not all patients have complete information.

\section{CONCLUSIONS}

In summary, to the best of our knowledge, the present comprehensive analysis of the overall 804 cases from SEER database is the first of its kind to clearly define the demographic features, clinicopathologic characteristics, treatment outcome and prognostic factors of patients with SFT, and is also the largest SFT series to date. Despite the above limitations, for the first time we found that the age $>51$ years $($ Ref- $\leq 51$ years, $\leq 53$ years for OS), SEER stage distant metastasized tumor (Reflocalized + regional tumor) and high pathologic grade III + IV (Ref- Grade I + II) were independent averse prognostic indicators for DFS and OS. Regarding the treatment, the surgery was an independent favorable prognosis factor for DFS (Ref- RT + surgery).

\section{MATERIALS AND METHODS}

\section{Date extraction}

SEER*Stat software from the National Cancer Institute (Surveillance Research Program, National Cancer Institute SEER*Stat software, http://www.seer.cancer.gov/ seerstat, Version 8.2.1) was applied for data extraction. International Classification of Diseases for Oncology (ICD-O-3) codes for hemangiopericytoma (9150) was used for identification of cases with a diagnosis of SFT registered in the SEER database.

\section{Variable selection and statistical analysis}

The study variables included gender, age, race, marital status, contract health service delivery areas (CHSDA) region, primary tumor location, SEER stage,
American joint committee on cancer (AJCC) stage, pathologic grade, treatment modalities, follow-up time and outcome status. Not all of the cases that we identified contained all these data. Collected data were analyzed using the software of the Statistical Package for Social Sciences, version 18.0, for Windows (SPSS, Chicago, IL). Differences in the numerical variables were assessed using the Student's test or non-parametric Wilcoxon test. The chi square test or Fisher exact test for categorical variables was used for two group comparisons of parameters. The survival curves were generated using the Kaplan-Meier method, and the log-rank test was performed to evaluate the survival difference. Adjusted hazard ratios (HRs) along with $95 \%$ confidence intervals (CIs) were calculated using the Cox proportional hazards regression model. When the $\mathrm{P}$ value was $<0.05$, the difference was regarded as statistically significant. All statistical tests were two tailed.

\section{ACKNOWLEDGMENTS}

The authors thank Kamila Abulimiti (MD) from Strayer University (USA) for her editorial help.

\section{FUNDING}

This study was supported by Project Funded by China Postdoctoral Science Foundation (2015M571493).

\section{CONFLICTS OF INTEREST}

The authors made no disclosures.

\section{REFERENCES}

1. Canter RJ, Beal S, Borys D, Martinez SR, Bold RJ and Robbins AS. Interaction of histologic subtype and histologic grade in predicting survival for soft-tissue sarcomas. J Am Coll Surg. 2010; 210:191-198. e192.

2. Aufforth RD, Baker JJ and Hong JK. Soft Tissue Sarcoma. Surgical Oncology. 2015.

3. Stout AP and Murray MR. Hemangiopericytoma A Vascular Tumor Featuring Zimmermann's Pericytes. Ann Surg. 1942; 116:26-33.

4. Brooks JS and Lee S. Contemporary diagnostics: sarcoma pathology update. J Surg Oncol. 2015; 111:513-519.

5. Fletcher CDM. The evolving classification of soft tissue tumours: an update based on the new WHO classification. Histopathology. 2006; 48.

6. Wushou A, Bai XF, Qi H, Xu Z, Zheng J and Li G. Haemangiopericytoma of the jaw. J Craniomaxillofac Surg. 2014; 42:689-694.

7. Wushou A, Miao XC and Shao ZM. Treatment outcome and prognostic factors of head and neck hemangiopericytoma: A meta-analysis. Head Neck. 2015; 37:1685-90. 
8. Enzinger FM and Smith BH. Hemangiopericytoma. An analysis of 106 cases. Hum Pathol. 1976; 7(1).

9. Mena H, Ribas JL, Pezeshkpour GH, Cowan DN and Parisi JE. Hemangiopericytoma of the central nervous system: a review of 94 cases. Hum Pathol. 1991; 22:84-91.

10. Espat NJ, Lewis JJ, Leung D, Woodruff JM, Antonescu CR, Shia J and Brennan MF. Conventional hemangiopericytoma. Cancer. 2002; 95:1746-1751.

11. Hall WA, Ali AN, Gullett N, Crocker I, Landry JC, Shu HK, Prabhu R and W C. Comparing central nervous system (CNS) and extra-CNS hemangiopericytomas in the Surveillance, Epidemiology, and End Results program: analysis of 655 patients and review of current literature. Cancer. 2012; 118:5331-5338.

12. Lahat G, Tuvin D, Wei C, Anaya DA, Bekele BN, Lazar AJ, Pisters PW, Lev D and Pollock RE. New perspectives for staging and prognosis in soft tissue sarcoma. Ann Surg Oncol. 2008; 15:2739-2748.

13. Soyuer S, Chang EL, Selek U, McCutcheon IE and Maor MH. Intracranial meningeal hemangiopericytoma: the role of radiotherapy. Cancer. 2004; 100:1491-1497.

14. Dufour H, Métellus P, Fuentes S, Murracciole X, Régis J, Figarella-Branger D and Grisoli F. Meningeal hemangiopericytoma: a retrospective study of 21 patients with special review of postoperative external radiotherapy. Neurosurgery. 2001; 48:756-763.

15. Combs SE, Thilmann C, Debus J and Schulz-Ertner D. Precision radiotherapy for hemangiopericytomas of the central nervous system. Cancer. 2005; 104:2457-2465.

16. Chang SD and Sakamoto GT. The role of radiosurgery for hemangiopericytomas. Neurosurg Focus. 2003; 14:1-5.

17. Sonabend AM, Zacharia BE, Goldstein H, Bruce SS, Hershman D, Neugut AI and Bruce JN. The role for adjuvant radiotherapy in the treatment of hemangiopericytoma: a Surveillance, Epidemiology, and End Results analysis: Clinical article. J Neurosurg. 2014; 120:300-308.

18. Melone AG, D'Elia A, Santoro F, Salvati M, Delfini R, Cantore $G$ and Santoro A. Intracranial hemangiopericytoma - our experience in 30 years: a series of 43 cases and review of the literature. World Neurosurg. 2014; 81:556-562.

19. Duval M, Hwang E and Kilty SJ. Systematic review of treatment and prognosis of sinonasal hemangiopericytoma. Head Neck. 2013; 35:1205-1210. 